\title{
THE PROGNOSTIC VALUE OF SOME INITIAL CLINICAL MANIFESTATIONS AND BIOCHEMICAL PARAMETERS FOR EVALUATING THE OUTCOME IN CORROSIVES POISONED CHILDREN
}

\author{
Eglal H. Elawady ${ }^{1}-$ Rabab N. Hafiz, ${ }^{1}$-Merhan A. Nasr ${ }^{2}$ \\ Departments of Forensic Medicine \&Clinical Toxicology ${ }^{1}$ and Radiology ${ }^{2}$ \\ Faculty of Medicine- Ain Shams University \\ ABSTRACT
}

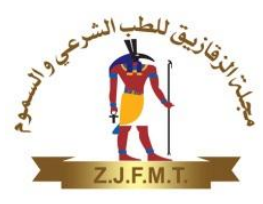

Corrosive products ingestions in children pose potentially devastating presentations and lifelong complications which remain one of the most challenging concerns encountered in medical practice. Prompt diagnosis and management are of outmost importance in decreasing mortality and achieving optimal long-term outcomes. Aim of the work: to investigate the predictability of the severity of corrosive poisoning outcome from some clinical findings and laboratory data at presentation. Patients and Methods: The study enrolled children with corrosive substances poisoning admitted to Poison Control Center-Ain Shams University during the period from January 2015 till September 2016. Demographic variables, on admission clinical findings, routine laboratory data and the outcome variables (length of hospital stay, development of stricture and mortality rate) were recorded. Patients were classified into two groups; the non-complicated group and the complicated group. Results: 106 patients, 56 males (52.8\%) with median age (3.06 2.57 years) met inclusion criteria. Signs and symptoms as (vomiting, dysphagia, drooling, hematemesis and respiratory distress) were found to be significantly higher in the complicated group as compared to the non-complicated group, while oral lesions and stridor showed insignificant difference between the two groups. Initial vomiting, drooling, dysphagia and hematemesis, but not respiratory distress, exhibited significant correlation with poor outcome (longer hospital stay, developing stricture/stenosis and increased mortality rate) together with high sensitivity and specificity prediction of stricture/stenosis formation 21 days post-ingestion (95.65\%-70\%, 91.49\%-55.56\%, 95.35\%-45.5\%, 92.86\%- 42.86\% respectively). Lower $\mathrm{pH}$ and $\mathrm{Hb}$ levels were more evident in the complicated group and were correlated with poor outcome. While, higher WBCs were only correlated with longer hospital stay time. Acidosis and anemia had significant sensitivity (91.30\%- $90.91 \%$ respectively) and specificity (44.43\%- $41.67 \%$ respectively) prediction of stricture/stenosis formation.

Conclusion: In children corrosive ingestion, some clinical manifestations as vomiting, drooling, dysphagia and hematemesis together with laboratory data as decreased $\mathrm{pH}$ and $\mathrm{Hb}$ levels and increased WBCs count on admission, were more obvious in the complicated cases and were found to be reliable predictors of outcome severity. Recommendations: It is recommended to carefully monitor initial signs and symptoms together with laboratory data to predict the outcome severity and avoid complications early enough.

Key words: corrosives, children, outcome, stricture.

Corresponding author: dreglalelawady@yahoo.com

\section{INTRODUCTION}

C orrosives ingestion by children is still a serious medical and social problem worldwide and especially in developing countries (Rafeey et al., 2016). In Egypt, caustic agents poisoning was considered one of the most encountered household products toxicities among children in a study done in Alexandria by Seif et al. (2016) and it represented the highest percentage after insecticides in a study by Maklad et al. (2012). Children represent $80 \%$ of corrosive injuries where corrosive ingestion is usually accidental 
compared to the suicidal and homicidal modes

Alkalis and acids are the two primary types of agents responsible for caustic exposures. Upon ingestion alkalis primarily damage the oropharynx and esophagus, whereas acids usually involve the distal part of esophagus and stomach (Sagar et al., 2016).

The characteristics and pathophysiology of tissue damage after caustic ingestion depends on its nature (alkali or acid), (liquid or solid), concentration, contact time, quantity and $\mathrm{pH}$ of the agent with $\mathrm{pH}<2$ and $>11$ being severer (Struck et al., 2016).

Ingestion of caustic agents leads to injuries to the gastrointestinal tract (GIT) and respiratory system with varying degrees from asymptomatic to devastating sequelae and/or death (Wightman et al., 2016). The most common late complications are esophageal strictures and stenosis, gastric stenosis of the antrum and pyloris, esophageal and stomach cancer (Vezakis et al., 2016).

Rapid assessment of the severity of caustic injury is very important because the prognosis depends on early application of appropriate treatment (Chen et al., 2003). There is a debate about performing early endoscopic examination as a tool of initial evaluation for patients with caustic ingestion especially in children, owing to high risks associated and the need for highly qualified health professionals. Endoscopy for corrosives ingestion is not a routine procedure in developing countries (Kaya et al., 2010; Urganci et al., 2014). Thus, there is an increasing need for evaluating and predicting the clinical course, hazards and long term complications using non-or less invasive low cost rapid measures. Also, to prevent unnecessary endoscopy and hospitalization, few studies have focused on the correlation between the clinical characteristics and the severity of corrosives injury (Kaya et al., 2010).

The aim of the study: is to evaluate the prognostic value of some initial signs and symptoms together with some biochemical parameters in predicting the outcome of corrosives poisoned children admitted to Poison in adults (Vezakis et al., 2016).

Control Center of Ain Shams University Hospitals (PCC-ASUH).

ETHICAL CONSIDERATIONS: followed ethical guidelines applied in the PCC that entail an informed written consent taken from his/her legal guardian in addition to head of PCC and ethical committee approval.

\section{PATIENTS AND METHODS}

The study enrolled children of both gender, admitted to PCC-ASUH in the period between the first of January 2015 and the end of September 2016, with acute corrosives poisoning based on history and clinical examination. On admission clinical evaluation during the first 24 hours was recorded. Inclusion criteria included: children less than 18 years old with single acute ingestion of corrosive substances. Exclusion criteria: adult patients, multiple ingestions, inconclusive history and referral with any intake of therapy. None of the patients underwent endoscopic examination for initial evaluation and classification due to technical and economical concerns.

Grouping: patients were classified into two groups; the complicated group which included any patient with one or more of: on admission hematemesis, melena, shock, signs and symptoms of esophageal or gastric perforation, respiratory distress, bronchopneumonia, pulmonary edema and/or acute respiratory distress syndrome (ARDS) and the non-complicated group: included patients with none of the aforementioned complications. The complicated group consisted of alkali corrosive ingestion (41 patients) and acid corrosive ingestion (28 patients).

\section{The Data:}

-Demographic variables: age, gender, type of caustic agent, amount and delay time.

-Clinical examination findings: vital data, signs and symptoms at presentation to the emergency department and complications (early and late).

\section{-Biochemical parameters:}


$>$ Serum electrolytes levels: sodium $\left(\mathrm{Na}^{+}\right)$ and potassium $\left(\mathrm{K}^{+}\right)$were determined by flame absorption photometer Jenway-PFP7 (Bibby Scientific Limited OSA, UK) (Rothrock et al., 1997).

$>$ Kidney function: serum creatinine and urea were measured by colorimetric method (Lawrence and Robert, 1993).

$\begin{array}{ll}\text { Liver enzymes: } & \text { aspartate } \\ \text { aminotransferase } & \text { (AST), }\end{array}$
aminotransferase (ALT) were determined by colorimetric method (Suber, 1994).

$>$ Random blood glucose level: done by calorimetric method (Kaplan, 1984).

$>$ Arterial blood gases: samples analyzed in the Blood Gas Analyzer ABL 800-Basic. The $\mathrm{pH}, \mathrm{PO} 2, \mathrm{PCO} 2$, and $\mathrm{HCO} 3$ values were recorded (Burnett et al., 1995).

$>$ Haematological tests: [Haemoglobin concentration $(\mathrm{Hb})$, total white blood cells (WBCs) count and platelet count] using coulter counter model M450 [coulter Electronics Ltd, Australia]. International normalized ratio (INR): using BE-Behnk Elektronik Coagulator.

$>$ Barium swallow imaging: was performed 21 days post-corrosive ingestion and results were interpreted according to Federle et al. (2004).

All patients received standard supportive medical treatment. This followed an approved standard protocol, which was dictated by the patient's clinical condition and included initial stabilization, preventing; neutralization, dilution and gastric emptying, providing; analgesics, H2 blockers, proton pump inhibitors, corticosteroids, antibiotics, good hydration, suitable balanced nutrition. The management also included X-ray imaging, admission in the inpatient wards or intensive care unit (ICU) and referral for surgery if indicated (Naik and Vadivelan, 2012).

-Outcome data: complete recovery, development of esophageal or gastric stricture/stenosis, duration of hospital stay and mortality rate.

Statistical analysis:
The results were statistically analyzed using the SPSS software, version 21 (SPSS, Inc., Chicago, IL).Quantitative data are described as Mean \pm SD and numbers (\%). Student's $t$-test was used to analyze the parametric data. Discrete variables were analyzed using Chi-square test $(\chi 2)$. Correlation coefficient was used to assess the relationship between variables and the outcome. Sensitivity, specificity, positive predictive value (PPV) and negative predictive value (NPP) were measured for the independent risk factors (predictors) of stricture/stenosis formation. $\mathrm{P}$ values $<0.05$ were considered statistically significant (Taylor, 1990).

\section{RESULTS}

One hundred and six patients, 56 males $(52.8 \%)$ and 50 females (47.2\%) met inclusion criteria. Mean age was (3.06 \pm 2.57 years). All cases were accidental ingestion of corrosive agents. The type of caustic agent was alkali in 57 patients $(53.77 \%)$ and acid in 49 patients (46.22\%). In the complicated group, there were 41 patients with alkali corrosive ingestion and 28 patients with acid corrosive ingestion. It was difficult to ascertain the exact ingested amount in each case, but it ranged apparently from 15 $\mathrm{ml}$ to $60 \mathrm{ml}$. The average delay time was $(6 \pm 4.28$ hours). Barium swallow imaging was performed in 56 patients; 11 in the noncomplicated group and 45 in the complicated group in the Radiology department of ASUH (Figures 1, 2 and 3). Seven patients died, all in the complicated group, with mortality rate $(6.6 \%)$.

Table (1): shows that there was non-significant difference between the non-complicated group and the complicated group regarding age, gender and delay time.

Table (2): shows that there was significant increase of heart rate and respiratory rate and significant decrease of systolic and diastolic blood pressure in the complicated group when compared to the non-complicated group, while temperature showed insignificant difference between the two groups.

The admission day clinical data of corrosive poisoned children; Table (3): shows that the 
most common finding was vomiting. Furthermore, there was significant increase in the complicated group in comparison to the non-complicated group regarding the incidence of (pallor, cyanosis, drooling of saliva, dysphagia, vomiting, hematemesis, and respiratory distress. While, there was nonsignificant increase of oropharyngeal lesions and stridor in the complicated group in comparison to the non-complicated group.

Table (4): shows that there was non-significant difference between alkali corrosive poisoned patients and acid corrosive poisoned patients in the complicated group regarding the incidence of admission day clinical manifestations (pallor, cyanosis, Oropharyngeal lesions, drooling of saliva, dysphagia, vomiting, hematemesis, stridor, and respiratory distress).

Table (5): shows that the most common complication encountered was hematemesis (35.84\%). While melena was (16\%), signs and symptoms of esophageal or gastric perforation $(3.77 \%)$, shock $(2.83 \%)$, respiratory distress $(19.8 \%)$, bronchopneumonia $(4.71 \%)$ and pulmonary edema/ARDS $(7.54 \%)$. There was non-significant difference between alkali corrosive poisoned patients and acid corrosive poisoned patients regarding the incidence of complications during hospital stay.

The biochemical parameters; Table (6): reveals that serum levels of $\mathrm{Na}^{+}, \mathrm{K}^{+}$, creatinine, urea, AST, ALT, glucose and INR showed nonsignificant difference between the noncomplicated group and the complicated group.

Table (7): shows that there was significant decrease of $\mathrm{pH}, \mathrm{PO}_{2}, \mathrm{PCO}_{2}$ and $\mathrm{HCO}_{3}$ in the complicated group as compared with the noncomplicated group.

Table (8): shows that there was significant decrease of $\mathrm{Hb}$ level, significant increase of WBCs count and non-significant increase of platelet count in the complicated group in comparison to the non-complicated group.

The outcome data; Table (9): reveals that there was significant decrease in the complete recovery rate and significant prolonged hospital stay time in the complicated group in comparison to the non-complicated group.
Among the 56 patients who underwent barium swallow examination, significant increase in stricture/stenosis formation was evident in the complicated group compared to the noncomplicated group. The mortality rate in the complicated group (10.14\%) was significant with the non-complicated group $(0 \%)$.

Table (10): Vomiting, hematemesis, drooling and dysphagia showed significant positive correlation, while, $\mathrm{pH}$ and $\mathrm{Hb}$ showed significant negative correlation with the outcome (hospital stay time, development of stricture/stenosis and mortality rate). WBCs count showed significant positive correlation with hospital stay time only. While, respiratory distress and platelet count did not show significant correlation with any of the outcome factors. For prediction of stricture/stenosis formation, vomiting showed (sensitivity $95.65 \%$, specificity $70 \%$, PPV $93.62 \%$ and NPV $77.78 \%$ ), hematemesis showed (sensitivity $92.86 \%$, specificity $42.86 \%$, PPV82.98\% and NPV 66.67\%), drooling showed (sensitivity 91.49\%, specificity $55.56 \%$, PPV 91.49\%and NPV 55.56\%), dysphagia showed (sensitivity $95.35 \%$, specificity $45.44 \%$, PPV $87.23 \%$ and NPV $71.43 \%$ ), pH showed (sensitivity 91.30\%, specificity $44.43 \%$, PPV $89.36 \%$ and NPV $50.01 \%$ ) and $\mathrm{Hb}$ showed (sensitivity $90.91 \%$, specificity $41.67 \%$, PPV $85.11 \%$ and NPV $55.56 \%$ ) (Table 11). 
Table (1): Student's t-test Statistical Analysis of age and delay time and Chi-square test $(\chi 2)$ of gender between non-complicated group and complicated group of corrosives poisoned patients.

\begin{tabular}{|l|l|l|l|l|}
\hline \multicolumn{1}{|c|}{ Groups } & $\begin{array}{l}\text { Non-complicated } \\
\text { group } \\
\text { (NO=37) }\end{array}$ & $\begin{array}{l}\text { Complicated group } \\
(\mathrm{NO}=69)\end{array}$ & $\mathrm{t}$ & $\mathrm{P}$ \\
\hline Aarameters & $2.81 \pm 1.8$ & $3.19 \pm 2.8$ & 0.83 & $>0.05$ \\
\hline Delay time (hours) (Mean \pm SD) $\mathfrak{a}$ & $6.02 \pm 3.8$ & $5.98 \pm 4.5$ & 0.04 & $>0.05$ \\
\hline $\begin{array}{l}\text { Gender (\%) \# } \\
\text { Male }\end{array}$ & $\begin{array}{l}20(54 \%) \\
\text { Female }\end{array}$ & $36(52.2 \%)$ & - & $>0.05$ \\
\hline
\end{tabular}

$\mathrm{P}>0.05$ non-significant difference. NO: Number. SD: standard deviation. a performed by Student's t-test Statistical Analysis.

\# performed by Chi-square test $\left(\chi^{2}\right)$.

Table (2): Student's t-test Statistical Analysis of vital signs between non complicated group and complicated group of corrosives poisoned patients.

$\mathrm{P}>0.05$ non-significant difference. ${ }^{*} \mathrm{P}<0.05$ significant difference. NO: Number. SD: standard deviation.

\begin{tabular}{|c|c|c|c|c|}
\hline Parameters & $\begin{array}{l}\text { Non-complicated } \\
\text { group } \\
(\mathrm{NO}=37) \\
\text { Mean } \pm \text { SD }\end{array}$ & $\begin{array}{l}\text { Complicated } \\
\text { group } \\
(\mathrm{NO}=69) \\
\text { Mean } \pm \mathrm{SD}\end{array}$ & $\mathrm{t}$ & $\mathrm{P}$ \\
\hline Heart rate (beat/minute) & $99.86 \pm 12.88$ & $109.68 \pm 14.67$ & 3.55 & $<0.05^{*}$ \\
\hline Systolic blood pressure (mmHg) & $102.97 \pm 7.4$ & $97.53 \pm 12.3$ & 2.83 & $<0.05^{*}$ \\
\hline Diastolic blood pressure ( $\mathrm{mmHg}$ ) & $66.21 \pm 8.28$ & $61.95 \pm 4.94$ & 2.86 & $<0.05^{*}$ \\
\hline Respiratory rate (breath/minute) & $19.21 \pm 2.63$ & $21.78 \pm 3.5$ & 4.23 & $<0.05^{*}$ \\
\hline Temperature ${ }^{0} \mathrm{C}$ & $37.03 \pm 0.15$ & $37.09 \pm 0.21$ & 1.61 & $>0.05$ \\
\hline
\end{tabular}


Table (3): Chi-square test $(\chi 2)$ of on admission clinical manifestations between non-complicated group and complicated group of corrosives poisoned patients.

\begin{tabular}{|c|c|c|c|}
\hline $\begin{array}{rr} & \text { Groups } \\
\text { Parameters } & \\
\text { NO }(\%) & \end{array}$ & $\begin{array}{l}\text { Non-complicated } \\
\text { group } \\
(\mathrm{NO}=37)\end{array}$ & $\begin{array}{l}\text { Complicated group } \\
(\mathrm{NO}=69)\end{array}$ & $\mathrm{P}$ \\
\hline Pallor & $4(10.81 \%)$ & $31(44.92 \%)$ & $<0.05^{*}$ \\
\hline Cyanosis & 0 & $8(11.59 \%)$ & $<0.05^{*}$ \\
\hline $\begin{array}{c}\text { Oropharyngeal lesion: } \\
\text { Edema } \\
\text { Burn } \\
\text { Ulcerations }\end{array}$ & $\begin{array}{l}17(45.94 \%) \\
0 \\
0\end{array}$ & $\begin{array}{l}22(31.88 \%) \\
2(2.89 \%) \\
3(4.34 \%)\end{array}$ & $\begin{array}{l}>0.05 \\
>0.05 \\
>0.05\end{array}$ \\
\hline Drooling of saliva & $10(27.02 \%)$ & $41(59.42 \%)$ & $<0.05^{*}$ \\
\hline Dysphagia & $21(56.75 \%)$ & $54(78.26 \%)$ & $<0.05^{*}$ \\
\hline Vomiting & $25(67.56 \%)$ & $61(88.4 \%)$ & $<0.05^{*}$ \\
\hline Hematemesis & 0 & $21(30.43 \%)$ & $<0.05^{*}$ \\
\hline Stridor/hoarseness of voice & $12(32.43 \%)$ & $34(49.27 \%)$ & $>0.05$ \\
\hline Respiratory distress \pm wheeze & 0 & $13(18.84 \%)$ & $<0.05^{*}$ \\
\hline
\end{tabular}

$\mathrm{P}>0.05$ non-significant difference. $* \mathrm{P}<0.05$ significant difference. NO: Number.

Table (4): Chi-square test $(\chi 2)$ of on admission clinical manifestations between alkali and acid corrosive ingestion in the complicated group of corrosives poisoned patients ( $\mathrm{NO}=69$ patients).

\begin{tabular}{|c|c|c|c|}
\hline $\begin{array}{r}\text { Parameters } \\
\text { NO }(\%)\end{array}$ & $\begin{array}{ll}\text { Alkali } & \text { corrosive } \\
\text { ingestion } & \\
(\mathrm{NO}=41) & \end{array}$ & $\begin{array}{l}\text { Acid corrosive ingestion } \\
(\mathrm{NO}=28)\end{array}$ & $\mathrm{P}$ \\
\hline Pallor & $19(46.34 \%)$ & $12(42.85 \%)$ & $>0.05$ \\
\hline Cyanosis & $5(12.19 \%)$ & $3(10.7 \%)$ & $>0.05$ \\
\hline $\begin{array}{c}\text { Oropharyngeal lesion: } \\
\text { Edema } \\
\text { Burn } \\
\text { Ulcerations } \\
\end{array}$ & $\begin{array}{l}13(31.7 \%) \\
1(2.4 \%) \\
2(4.8 \%)\end{array}$ & $\begin{array}{l}9(32.1 \%) \\
1(3.5 \%) \\
1(3.5 \%) \\
\end{array}$ & $\begin{array}{l}>0.05 \\
>0.05 \\
>0.05\end{array}$ \\
\hline Drooling of saliva & $23(56 \%)$ & $18(63.2 \%)$ & $>0.05$ \\
\hline Dysphagia & $31(75.6 \%)$ & $23(82.1 \%)$ & $>0.05$ \\
\hline Vomiting & $36(87.8 \%)$ & $25(89.2 \%)$ & $>0.05$ \\
\hline Hematemesis & $9(21.9 \%)$ & $12(42.8 \%)$ & $>0.05$ \\
\hline Stridor/hoarseness of voice & $19(46.34 \%)$ & $15(53.5 \%)$ & $>0.05$ \\
\hline Respiratory distress \pm wheeze & $8(19.5 \%)$ & $5(17.8 \%)$ & $>0.05$ \\
\hline
\end{tabular}

P > 0.05 non-significant difference. NO: Number. 
Table (5): Chi-square test $(\chi 2)$ of complications in corrosives poisoned patients during hospital stay.

\begin{tabular}{|l|l|l|l|l|}
\hline $\begin{array}{l}\text { Complications } \\
\text { NO }(\%)\end{array}$ & $\begin{array}{l}\text { Alkali } \\
\text { corrosive } \\
\text { ingestion } \\
\text { (NO=57) }\end{array}$ & $\begin{array}{l}\text { Acid corrosive } \\
\text { ingestion } \\
\text { (NO=49) }\end{array}$ & $\mathrm{P}$ & $\begin{array}{l}\text { Total } \\
(\mathrm{NO}=106)\end{array}$ \\
\hline Hematemesis & $18(31.5 \%)$ & $20(40.8 \%)$ & $>0.05$ & $38(35.8 \%)$ \\
\hline Melena & $8(14 \%)$ & $9(18.3 \%)$ & $>0.05$ & $17(16 \%)$ \\
\hline Shock & $1(1.7 \%)$ & $2(4 \%)$ & $>0.05$ & $3(2.83 \%)$ \\
\hline $\begin{array}{l}\text { Signs and symptoms of esophageal or } \\
\text { gastric perforation }\end{array}$ & $3(5.2 \%)$ & $1(2 \%)$ & $>0.05$ & $4(3.77 \%)$ \\
\hline Respiratory distress & $12(21 \%)$ & $9(18.3 \%)$ & $>0.05$ & $21(19.8 \%)$ \\
\hline Bronchopneumonia & $3(5.2 \%)$ & $2(4 \%)$ & $>0.05$ & $5(4.71 \%)$ \\
\hline Pulmonary edema/ARDS & $5(8.7 \%)$ & $3(6.1 \%)$ & $>0.05$ & $8(7.54 \%)$ \\
\hline
\end{tabular}

$\mathrm{P}>0.05$ non-significant difference. NO: Number. ARDS: acute respiratory distress syndrome.

Table (6): Student's t-test Statistical Analysis of serum levels; sodium $\left(\mathrm{Na}^{+}\right)$, potassium $\left(\mathrm{K}^{+}\right)$, creatinine, urea, aspartate aminotransferase (AST), alanine aminotransferase (ALT), glucose and international normalized ratio (INR) between non-complicated group and complicated group of corrosives poisoned patients.

P > 0.05 non-significant difference. NO: Number. SD: standard deviation.

\begin{tabular}{|c|c|c|c|c|}
\hline $\begin{array}{c}\text { Groups } \\
\text { Parameters }\end{array}$ & $\begin{array}{c}\text { Non-complicated } \\
\text { group } \\
(\mathrm{NO}=37) \\
\text { Mean } \pm \mathrm{SD}\end{array}$ & $\begin{array}{c}\text { Complicated } \\
\text { group } \\
(\mathrm{NO}=69) \\
\text { Mean } \pm \mathrm{SD}\end{array}$ & $\mathrm{t}$ & $\mathrm{P}$ \\
\hline $\mathrm{Na}^{+}(\mathrm{mEq} / \mathrm{L})$ & $134.05 \pm 6.35$ & $135.01 \pm 11$ & 0.56 & $>0.05$ \\
\hline $\mathrm{K}^{+}(\mathrm{mEq} / \mathrm{L})$ & $3.64 \pm 0.34$ & $3.5 \pm 0.49$ & 1.71 & $>0.05$ \\
\hline S. creatinine $(\mathrm{mg} / \mathrm{dL})$ & $0.65 \pm 0.11$ & $0.63 \pm 0.16$ & 0.8 & $>0.05$ \\
\hline Urea $(\mathrm{mg} / \mathrm{dL})$ & $20.91 \pm 2.99$ & $19.21 \pm 6.92$ & 1.75 & $>0.05$ \\
\hline AST (IU/L) & $20.45 \pm 3.9$ & $24.4 \pm 11.6$ & 0.96 & $>0.05$ \\
\hline ALT (IU/L) & $20.73 \pm 3.71$ & $21.72 \pm 5.32$ & 0.68 & $>0.05$ \\
\hline Glucose (mg/dL) & $102.89 \pm 45.35$ & $94.01 \pm 56.46$ & 0.87 & $>0.05$ \\
\hline INR & $1.03 \pm 0.05$ & $1.05 \pm 0.05$ & 1.37 & $>0.05$ \\
\hline
\end{tabular}

Table (7): Student's t-test Statistical Analysis of arterial blood gas (ABG) values: $\mathrm{pH}$, partial pressure of oxygen $\left(\mathrm{PO}_{2}\right)$, partial pressure of carbon dioxide $\left(\mathrm{PCO}_{2}\right)$ and bicarbonate $\left(\mathrm{HCO}_{3}\right)$ between noncomplicated group and complicated group of corrosives poisoned patients

\begin{tabular}{|c|c|c|c|c|}
\hline $\begin{array}{c}\text { Groups } \\
\text { Parameters }\end{array}$ & $\begin{array}{c}\text { Non-complicated } \\
\text { group } \\
(\mathrm{NO}=37) \\
\text { Mean } \pm \mathrm{SD}\end{array}$ & $\begin{array}{c}\text { Complicated } \\
\text { group } \\
(\mathrm{NO}=69) \\
\text { Mean } \pm \mathrm{SD}\end{array}$ & $\mathrm{t}$ & $\mathrm{P}$ \\
\hline $\mathrm{pH}$ & $7.37 \pm 0.03$ & $7.33 \pm 0.06$ & 4.81 & $<0.05^{*}$ \\
\hline $\mathrm{PO}_{2}(\mathrm{mmHg})$ & $74.61 \pm 8.56$ & $62.1 \pm 11.8$ & 6.25 & $<0.05^{*}$ \\
\hline $\mathrm{PCO}_{2}(\mathrm{mmHg})$ & $40.03 \pm 4.69$ & $37.72 \pm 5.88$ & 2.2 & $<0.05^{*}$ \\
\hline $\mathrm{HCO}_{3}(\mathrm{mEq} / \mathrm{L})$ & $20.97 \pm 1.14$ & $19.27 \pm 2.58$ & 4.67 & $<0.05^{*}$ \\
\hline
\end{tabular}

$* \mathrm{P}<0.05$ significant difference. NO: Number. SD: standard deviation. 
Table (8): Student's t-test Statistical Analysis of hematological parameters [hemoglobin ( $\mathrm{Hb}$ ) level, total white blood cells count (WBCs) and platelet counts] between non-complicated group and complicated group of corrosives poisoned patients.

\begin{tabular}{|l|l|l|l|l|}
\hline \multicolumn{1}{|c|}{ Groups } & \multicolumn{1}{|c|}{$\begin{array}{c}\text { Non-complicated } \\
\text { group } \\
\text { Parameters }\end{array}$} & $\begin{array}{c}\text { Complicated } \\
\text { group } \\
\text { Mean } \pm \text { SD }\end{array}$ & $\begin{array}{c}\text { MO=69) } \\
\text { Mean } \pm \text { SD }\end{array}$ & $\mathrm{t}$ \\
\hline $\mathrm{Hb}(\mathrm{g} / \mathrm{dl})$ & $11.45 \pm 1.08$ & $9.71 \pm 1.97$ & 5.84 & $<0.05^{*}$ \\
\hline $\mathrm{WBCs}\left(\mathrm{x} 10^{3} / \mathrm{mm}^{3}\right)$ & $10.41 \pm 3.64$ & $12.06 \pm 3.99$ & 2.14 & $<0.05^{*}$ \\
\hline Platelet $\left(\mathrm{x} 10^{3} / \mathrm{mm}^{3}\right)$ & $307.91 \pm 101.04$ & $350.67 \pm 92.51$ & 1.87 & $>0.05$ \\
\hline
\end{tabular}

$\mathrm{P}>0.05$ non-significant difference. $* \mathrm{P}<0.05$ significant difference. NO: Number. SD: standard deviation.

Table (9): Outcome data among the non-complicated group and complicated group of corrosives poisoned patients.

\begin{tabular}{|c|c|c|c|c|}
\hline Parameters & $\begin{array}{l}\text { Non-complicated } \\
\text { group } \\
(\mathrm{NO}=37)\end{array}$ & $\begin{array}{l}\text { Complicated } \\
\text { group } \\
(\mathrm{NO}=69)\end{array}$ & $\mathrm{t}$ & $\mathrm{P}$ \\
\hline Recovery: NO (\%) \# & $33(89.18 \%)$ & $19(27.53 \%)$ & - & $<0.05^{*}$ \\
\hline \multirow{2}{*}{$\begin{array}{l}\text { Barium swallow results: } \\
\text { Stricture/stenosis formation (-) \# } \\
\text { Stricture/stenosis formation (+) \# }\end{array}$} & $(\mathrm{NO}=11)$ & $(\mathrm{NO}=45)$ & \multirow[t]{2}{*}{-} & \\
\hline & $\begin{array}{l}7(63.63 \%) \\
4(36.36 \%)\end{array}$ & $\begin{array}{l}2(4.44 \%) \\
43(95.55 \%)\end{array}$ & & $\begin{array}{l}<0.05^{*} \\
<0.05^{*}\end{array}$ \\
\hline Hospital stay time (days)(Mean \pm SD) $\mathfrak{a}$ & $3.2 \pm 2.2$ & $10.34 \pm 9.57$ & 5.54 & $<0.05^{*}$ \\
\hline \multirow[t]{2}{*}{ Death: NO (\%) \# } & 0 & $7(10.14 \%)$ & \multirow[t]{2}{*}{-} & \multirow[t]{2}{*}{$<0.05^{*}$} \\
\hline & $(6.6 \%)$ & & & \\
\hline
\end{tabular}

*P $<0.05$ significant difference. NO: Number. SD: standard deviation. $₫$ performed by Student's t-test Statistical Analysis.

\# performed by Chi-square test ( $\chi 2)$. (-) absent (+) present.

Table (10): Correlation of some clinical findings and biochemical results with the outcome (hospital stay time, development of visceral stricture/stenosis and mortality rate) in corrosive poisoned patients.

\begin{tabular}{|l|l|l|l|l|l|l|}
\hline \multirow{2}{*}{} & \multicolumn{2}{l}{$\begin{array}{l}\text { Hospital stay time } \\
\text { (days) }\end{array}$} & \multicolumn{2}{l|}{$\begin{array}{l}\text { Stricture/stenosis } \\
\text { formation }\end{array}$} & \multicolumn{2}{l|}{ Mortality rate } \\
\cline { 2 - 8 } & $\mathrm{r}$ & $\mathrm{P}$ & $\mathrm{r}$ & $\mathrm{P}$ & $\mathrm{r}$ & $\mathrm{P}$ \\
\hline Vomiting & 0.71 & $<0.05^{*}$ & 0.74 & $<0.05^{*}$ & 0.81 & $<0.05^{*}$ \\
\hline Hematemesis & 0.54 & $<0.05^{*}$ & 0.52 & $<0.05^{*}$ & 0.47 & $<0.05^{*}$ \\
\hline Drooling & 0.57 & $<0.05^{*}$ & 0.61 & $<0.05^{*}$ & 0.45 & $<0.05^{*}$ \\
\hline Dysphagia & 0.7 & $<0.05^{*}$ & 0.54 & $<0.05^{*}$ & 0.65 & $<0.05^{*}$ \\
\hline Respiratory distress & 0.18 & $>0.05$ & 0.13 & $>0.05$ & 0.19 & $>0.05$ \\
\hline pH & -0.29 & $<0.05^{*}$ & 0.64 & $<0.05^{*}$ & 0.53 & $<0.05^{*}$ \\
\hline Hb & -0.32 & $<0.05^{*}$ & 0.53 & $<0.05^{*}$ & 0.48 & $<0.05^{*}$ \\
\hline WBCs & 0.35 & $<0.05^{*}$ & 0.11 & $>0.05$ & 0.03 & $>0.05$ \\
\hline Platelet & 0.06 & $>0.05$ & 0.16 & $>0.05$ & 0.02 & $>0.05$ \\
\hline
\end{tabular}

Hb: hemoglobin. WBCs: whole blood cells. $r$ correlation coefficient. P> 0.05 non significant difference. $* \mathrm{P}<0.05$ significant difference. 
Table (11): Sensitivity, specificity, positive predictive value (PPV) and negative predictive value (NPV) of on admission vomiting, hematemesis, drooling of saliva, dysphagia, $\mathrm{pH}$ and $\mathrm{Hb}$ (hemoglobin) levels for the prediction of visceral stricture/stenosis development 21 days post ingestion in corrosive poisoned patients.

\begin{tabular}{|l|l|l|l|l|}
\hline & Sensitivity & Specificity & PPV & NPP \\
\hline Vomiting & $95.65 \%$ & $70 \%$ & $93.62 \%$ & $77.78 \%$ \\
\hline Hematemesis & $92.86 \%$ & $42.86 \%$ & $82.98 \%$ & $66.67 \%$ \\
\hline Drooling & $91.49 \%$ & $55.56 \%$ & $91.49 \%$ & $55.56 \%$ \\
\hline Dysphagia & $95.35 \%$ & $45.44 \%$ & $87.23 \%$ & $71.43 \%$ \\
\hline pH & $91.30 \%$ & $44.43 \%$ & $89.36 \%$ & $50.01 \%$ \\
\hline $\mathrm{Hb}$ & $90.91 \%$ & $41.67 \%$ & $85.11 \%$ & $55.56 \%$ \\
\hline
\end{tabular}
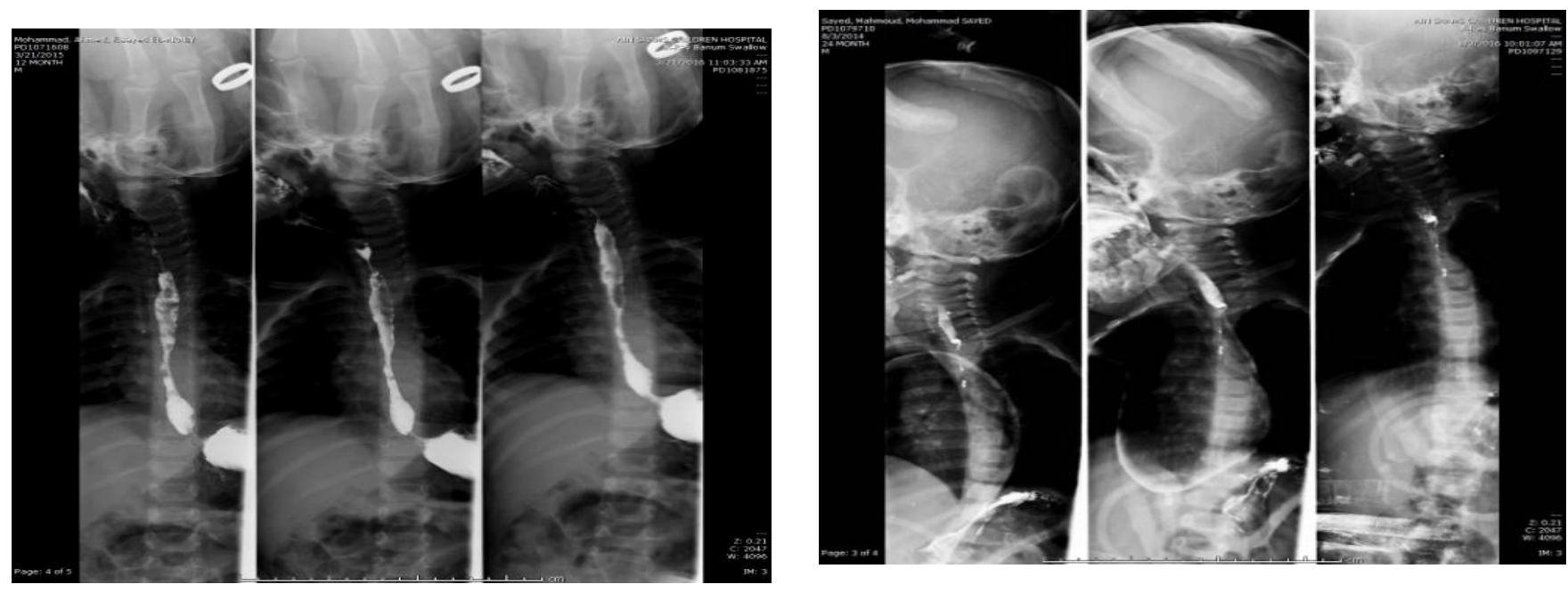

Figure (1): Barium swallow 21 days after alkali [potash (potassium hydroxide/KOH)] ingestion in a 1 year-old male patient showing middle third esophogeal stricture.
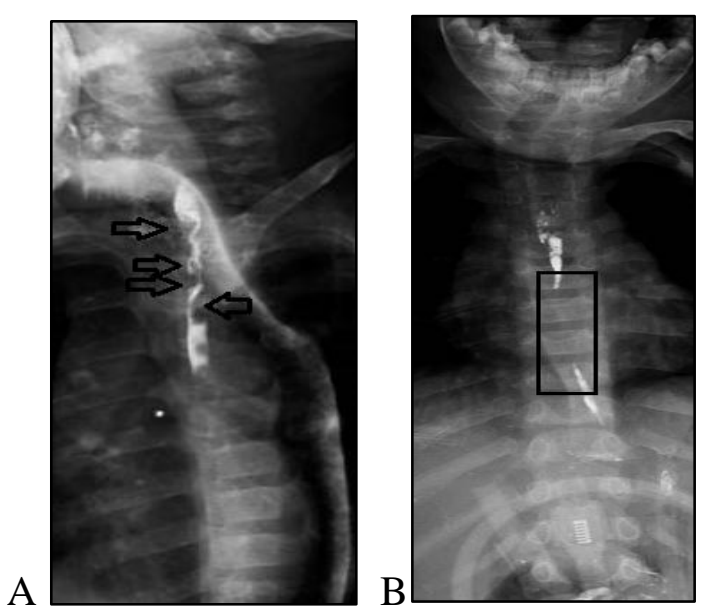

Figure (2): Barium swallow 21 days after alkali [potash (potassium hydroxide/KOH)] ingestion in a 2 year-old male patient showing long esophogeal stricture.

Figure (3): Barium swallow 21 days after acid [sulfuric acid (H2SO4)] ingestion in a 4 year-old male patient [A] Oblique view revealed multiple small strictures related to the upper intra thoracic course of the esophagus. [B] AP view of the same child revealed also long stricture involving the lower third of esophagus 


\section{DISCUSSION}

The potentially catastrophic presentation and lifelong complications that result from corrosive ingestion make it one of the most challenging clinical situations (Sudarsi et al., 2015). Children especially boys younger than 5 years of age are at high risk because of their natural curiosity, efforts to gain autonomy and lack of awareness of their surroundings dangers, together with easy accessibility of household solutions of drain cleaners containing sodium hydroxide $(\mathrm{NaOH})$ and/or potassium hydroxide $(\mathrm{KOH})$ which are the principal causes of corrosive GIT injury. Also, toilet bowl cleaners, detergents or bleach are common ingested caustic materials (Daradka, 2005; Urganci et al., 2014).

Prompt diagnosis and management are of crucial importance in reducing mortality and achieving optimal long-term outcomes (Vezakis et al., 2016). In children, it is often difficult to distinguish between suspected and confirmed cases of ingestion and a uniform inclusion criterion for performing an endoscopy is still debatable. Thus it is important to utilize signs and symptoms to identify patients who may or may not need diagnostic or therapeutic measures or be prone to poor outcome (Betalli et al., 2008; Urganci et al., 2014).

In this study the presence of some on admission signs and symptoms as (vomiting, dysphagia, drooling, hematemesis and respiratory distress) were found to be significantly higher in the complicated group as compared to the non-complicated group, while there was insignificant difference between the two groups regarding oral lesions and stridor. Initial vomiting, drooling, dysphagia and hematemesis, but not respiratory distress, were found to be significantly correlated with poor outcome (longer hospital stay, developing stricture/stenosis and increased mortality rate) together with high sensitivity and specificity prediction of stricture/stenosis formation 21 days post-ingestion $(95.65 \%-70 \%, 91.49 \%$ $55.56 \%, \quad 95.35 \%-45.5 \%, \quad 92.86 \%-\quad 42.86 \%$ respectively). Moreover there was nonsignificant difference between alkali corrosive poisoned patients and acid corrosive poisoned patients in the complicated group regarding the incidence of admission day clinical manifestations. Similar to the findings in the current study, vomiting was the most common symptom $44.7 \%$ in a study by Demirören et al. (2015). Together with dysphagia, both were found to be strongly related to increased morbidity and mortality (Sagar et al., 2016).

The relationship between symptoms and severity of injury is uncertain. Drooling of saliva and stridor were considered $100 \%$ specific for significant esophageal injury in a study by Contini and Scarpignato (2013). Earlier studies have suggested that the presence of two or more clinical findings of the triad (vomiting, drooling and stridor) in patients with a history of caustic substance ingestion may be a reliable predictor of esophageal injury (Crain et al., 1984; Lamireau et al., 2001). Gupta et al. (2001) noticed that all patients with clinically significant (second-degree and thirddegree) esophageal injury were symptomatic at initial assessment. Also, Chen et al. (2003) believe that patients who present with 2 or more of signs and symptoms after ingesting caustic agents are associated with increased risk of complications and esophageal stricture. Vomiting and drooling of saliva were found to be strongly correlated with severe esophageal lesions, while minor lesion on endoscopic examination evolved later into serious esophageal injury (Kaya et al., 2010). This can be explained by the fact that vomiting results in re-exposure of esophagus to the caustic agent with additional injuries and aspiration hazards.

Prolonged dysphagia and drooling (1224 hours) predicted esophageal scar formation with $100 \%$ sensitivity and $90.1 \%$ specificity (Nuutinen et al., 1994). Bonnici et al. (2014) supported the fact that any evidence of vomiting, drooling, dysphagia or pain clearly indicated the need for an endoscopy with greater caution in children due to the likelihood of significant damage of upper GIT.

Hematemesis is strongly related to decreased hemoglobin $(\mathrm{Hb})$ level in corrosive poisoning (Rao et al., 2014). In the present 
study, both were found to be correlated with longer hospital stay, likelihood of stricture/stenosis formation and mortality rate with sensitivity $(92.86 \%-90.91 \%)$ and specificity $(42.86 \%-41.67 \%)$ respectively. Hematemesis, like vomiting, dysphagia and drooling, could be nonspecific warning of later esophageal stenosis and stricture, yet, they are not necessarily correlated with extent of early esophageal injury (Gharib et al., 2016). In a study by Sudarsi et al. (2015) 16 patients of twenty five with haemetemesis had severe injury of esophagus and stomach even in the absence of oropharyngeal lesion. Betalli et al. (2008) believed that the risk of severe damage increases proportionally with the number of signs and symptoms and considered hematemesis naturally one of the most obvious predictors of the presence of severe lesions.

Regarding respiratory distress, it can be the result of aspiration of the caustic substances into trachea-bronchial tree with subsequent respiratory insult, or due to hyperventilation related to the degree of acidosis present (Dalus et al., 2013). Some authors believe respiratory distress to be highly predictive of increased morbidity (Lamireau et al. 2001).

On the other hand, studies showed that $70 \%$ of patients with severe oropharyngeal lesions did not have significant esophageal or gastric post-corrosive burns. Therefore, injuries of the oropharynx are not a reliable indicator for the eventual damage to the esophagus or stomach (Chibishev et al., 2012; Gharib et al., 2016).

In contrast to this work, several studies have indicated that clinical manifestations of corrosives ingestion are poor predictors of the degree and depth of esophageal or gastric injury and that signs and symptoms do not necessarily correlate with the severity of corrosive poisoning (Lupa et al., 2009; Boskovic and Stankovic, 2014; Sudarsi et al., 2015). They concluded that absence of any clinical findings does not rule out a severe esophageal or gastric injury.

Consistent with the literature, few studies have verified the predictive value of laboratory tests for corrosives morbidity (Kaya et al., 2010). In the present study, there was non-significant decrease of $\mathrm{K}^{+}$level in the complicated group compared to noncomplicated group. In accordance, prior studies revealed that serum electrolytes are not clearly indicatives of corrosives bad prognosis (Otcu et al., 2003). The lower level of $\mathrm{K}^{+}$in the complicated group in this study can be attributed to vomiting.

Hematemesis, vomiting and third space sequestration are the main causes of hypovolemia and acute circulatory compromise in corrosive poisoning especially in children (Naik and Vadivelan 2012). This goes hand in hand with the results of this study of relative decrease of blood pressure and increase of heart rate in the complicated group as compared to the non-complicated group. Hypovolemia and dehydration leads to conditions of anaerobic metabolism and acidosis which is exacerbated by hypoperfusion and hypoxia. Significant acidic ingestions may also induce metabolic acidosis (Dalus et al., 2013). Low $\mathrm{pH}$ in the current study was evident in the complicated group compared to the non-complicated group and was correlated with poor outcome with sensitivity $(91.3 \%)$ and specificity $(44.43 \%)$ for development of stricture/stenosis 21 days postingestion. Similarly, metabolic acidosis especially with $\mathrm{pH}$ less than 7.22 indicates tissue necrosis and significant damage (Gharib et al., 2016). Naik and Vadivelan (2012) stated that arterial blood $\mathrm{pH}$ and base deficit correlate with severity and adverse outcomes. Metabolic acidosis and hematemesis, either at presentation or as a complication, had significant association with mortality and development of caustic stricture (Dalus et al., 2013; Vezakis et al., 2016).

Regarding WBCs, significant higher WBCs count was found in the complicated group in comparison to the non-complicated group in the current work. Higher WBCs count only correlated with longer hospital stay time. Similar to these findings, Leukocytosis, was observed within 24 hours of presentation in $57.5 \%$ of the corrosive poisoned patients in a 
study by Dalus et al. (2013). Kaya et al. (2010) stated that although presence of high WBCs count was found significantly more frequent in children with high grade corrosive injury, it was not shown to be a sensitive predictor. Conversely, Naik and Vadivelan (2012) verified that WBCs count $>20,000 / \mathrm{mm}^{3}$ is an independent predictor of mortality in corrosive poisoning. Also, few studies have revealed a significant correlation between WBCs count and organ injury (Rigo et al., 2002; Chen et al., 2003; Havanond and Havanond, 2007). Leukocyte count is a useful parameter for monitoring inflammation (Duyar et al., 2016). Its level is usually used in evaluating the severity of tissue injury and was found to correlate with degree of early visceral damage (Chen et al., 2003). One of the possible mechanisms of elevated WBCs count in caustic toxicity is mobilization of the leukocyte marginal pool as an inflammatory response to the corrosive tissue injury. Another mechanism could be WBCs positive correlation with bacterial translocation. However, seldom experimental studies have shown an infection in corrosive esophageal burns (Kiyan et al., 2004; Ozel et al., 2004). The explanation might be that WBCs could be useful in evaluating the severity of acute esophageal or gastric injury, morbidities and other co-exiting inflammatory conditions (eg. Pneumonitis, bronchopneumonia, etc..) which eventually necessitate longer hospital stay time, but was not a valuable indicator for later caustic stricture formation and mortality rate.

Likewise, increased platelet count has recently gained a significant importance as a marker in inflammation and immunity, besides being major elements of hemostasis (Coşkun et al., 2014). In the current study insignificant increase of platelet count was observed in the complicated group in comparison to the noncomplicated group and no correlation with the outcome was noticed regarding platelet count.

\section{CONCULOSION}

Corrosive injury is still a major pediatric emergency among young children. It carries a major risk of complications. The study demonstrated that clinical features particularly vomiting, hematemesis, drooling of saliva and dysphagia together with biochemical parameters namely $\mathrm{pH}, \mathrm{Hb}$ and WBCs are good predictors of outcome severity in corrosive poisoned children and can help estimate the degree of morbidity and mortality, and consequently guide the management (by identifying patients who are at high risk to be early admitted to ICU without delay in the inpatient wards, deciding suitable feeding protocols and by strongly recommending for early endoscopy in equipped places and accordingly, timely surgical intervention will proceed).

\section{RECOMMENDATIONS}

It is recommended to carefully monitor signs and symptoms together with laboratory data in developing countries where endoscopy and other techniques, for early evaluation of severity, are risky, lacked or not readily available. Preventive measures (safe containers' packing, labeling, out of children's sight and reach, lower concentrations of caustics) will always remain the main recommendation. Further research into the prognostic validity of other clinical and laboratory findings on larger number and longer periods is suggested.

\section{REFERENCES}

Betalli, P.; Falchetti, D.; Giuliani, S.; et al., (2008): Caustic ingestion in children: is endoscopy always indicated? The results of an Italian multicenter observational study. Gastrointestinal Endoscopy; 68(3): 434- 439.

Bonnici, K.; Wood, D. and Dargan, P. (2014):

Should computerized tomography replace endoscopy in the evaluation of symptomatic ingestion of corrosive substances? Clin Toxicol (Phila);52(9):911-925.

Boskovic, A. and Stankovic, I. (2014): Predictability of gastroesophageal caustic injury from clinical findings: is endoscopy mandatory in children? Eur J Gastroenterol Hepatol;26(5):499-503.

Burnett, R.; Covington, A.; Fogh-Anderson, N.; et al., (1995): Approved FCC recommendations on whole blood sampling, transport and storage for simultaneous 
determination of $\mathrm{pH}$ blood gases and electrolytes. Eur J Chem Clin Biochem; 33: 247-253.

Chen, T.; Ko, S.; Chuang, J.; et al., (2003): Predictors of esophageal stricture in children with unintentional ingestion of caustic agents. Chang Gung Med J;26:233-239.

Chibishev, A.; Pereska, Z.; Chibisheva, V.; et al., (2012): Corrosive poisonings in adults. mater sociomed.; 24(2): 125-130.

Contini, S. and Scarpignato, C. (2013): Caustic injury of the upper gastrointestinal tract: A comprehensive review. World J Gastroenterol; 19(25): 3918-3930.

Coşkun, B.; Öksüz, M.; Ermurat, S.; et al., (2014): Neutrophil lymphocyte ratio can be a valuable marker in defining disease activity in patients who have started antitumor necrosis factor (TNF) drugs for ankylosing spondylitis. Eur J Rheumatol; 1: 101-105.

Crain, E.; Gershel, J. and Mezey, A. (1984): Caustic ingestions: Symptoms as predictors of esophageal injury. Am J Dis Child;138:863865.

Dalus, D.; Mathew, A. and Somarajan, P. (2013): Formic acid poisoning in a tertiary care center in South India: A 2-year retrospective analysis of clinical profile and predictors of mortality. J Emerg Med;44(2):373-380.

Daradka, I. (2005): Esophageal injury: a study of caustic ingestion in 83 children. Jordan medical journal;39(2): 149-195.

Demirören, K.; Kocamaz, H. and Doğan, Y. (2015): Gastrointestinal system lesions in children due to the ingestion of alkali and acid corrosive substances. Turk J Med Sci;45(1):184-190.

Duyar, S.; Solak, Y.; Tekis, D.; et al., (2016): Platelet to lymphocyte ratio as a novel prognostic marker in male patients with chronic obstructive pulmonary disease. Int $\mathbf{J}$ Respir Pulm Med; 3:043.

Federle, M.; Jeffrey, R.; Anne, V.; et al., (2004): Caustic esophagitis. In: Diagnostic Imaging Abdomen $1^{\text {st }}$ edition. Amirsys pub. Canada, PP: 2-24.
Gharib, B.; Mohammadpour, M.; Yaghmaie, B.; et al., (2016): Caustic agent ingestion by a 1.5-year-old boy. Acta Med Iran; 54(7):465470.

Gupta, S.; Croffie, J. and Fitzgerald, J. (2001): Is esophagogastroduodenoscopy necessary in all caustic ingestions? J Pediatr Gastroenterol Nutr; 32:50-3.

Havanond, C. and Havanond, P. (2007): Initial signs and symptoms as prognostic indicators of severe gastrointestinal tract injury due to corrosive ingestion. J Emerg Med;33:349-353.

Kaplan, L. (1984): Glucose. In: Clinical Chemistry, Louis S (ed.) Mosby Co. Toronto; PP: 1032-1036.

Kaya, M.; Özdemir, T.; Sayan, A.; et al., (2010): The relationship between clinical findings and esophageal injury severity in children with corrosive agent ingestion. Turkish Journal of Trauma and Emergency Surgery; 16(6):537-540.

Kiyan, G.; Aktas, S.; Ozel, K.; et al., (2004): Effects of hyperbaric oxygen therapy on caustic esophageal injury in rats. J Pediatr Surg;39:1188-1193.

Lamireau, T.; Rebouissoux, L.; Denis, D.; et al., (2001): Accidental caustic ingestion in children: is endoscopy always mandatory? J Pediatr Gastroenterol Nutr; 33:81-84.

Lawrence, M. and Robert, H. (1993): Methods of determination of blood urea nitrogen, urea and serum creatinine. In: Tietz Textbook of Clinical Chemistry, $2^{\text {nd }}$ edition, WB Saunders Company, London; PP: 621.

Lupa, M.; Magne, J. and Amedee, R. (2009): Update on the diagnosis and treatment of caustic ingestion. Ochsner J; 9(2): 54-59.

Maklad, A.; Emara, A.; El-Maddah, E.; et al., (2012): Pediatric poisoning in Egypt. Journal of Applied Pharmaceutical Science; 2(02): 0106.

Naik, R. and Vadivelan, M. (2012): Corrosive poisoning. Indian Journal of Clinical Practice; 23: (3):131-134.

Nuutinen, M.; Uhari, M.; Karvali, T.; et al., (1994): Consequences of caustic ingestions in children. Acta Paediatr; 83(11):1200-5. 
Otcu, S.; Karnak, I.; Tanyel, F.; et al., (2003):

Biochemical indicators of caustic ingestion and/or accompanying esophageal injury in children. Turk J Pediat; 45:21-5.

Ozel, S.; Dagli, T.; Yuksel, M.; et al., (2004):

The roles of free oxygen radicals, nitric oxide, and endothelin in caustic injury of rat esophagus. J Pediatr Surg; 39:1381-1385.

Rafeey, M.; Ghojazadeh, M.; Sheikhi, S.; et al., (2016): Caustic ingestion in children: a systematic review and meta-analysis. J Caring Sci; 5(3):251-265.

Rao, M.; Rathi, A.; Reddy, S.; et al., (2014): Intercostal artery pseudoaneurysm complicating corrosive acid poisoning: Diagnosis with CT and treatment with transarterial embolisation. Indian $\mathrm{J}$ Radiol Imaging; 24(2): 135-138.

Rigo, G.; Camellini, L.; Azzolini, F.; et al., (2002): What is the utility of selected clinical and endoscopic parameters in predicting the risk of death after caustic ingestion? Endoscopy; 34:304-310.

Rothrock, S.; Green, S.; McArthur, C.; et al., (1997): Detection of electrolyte abnormalities in children presenting to the emergency department: a multicenter, prospective analysis. Detection of electrolyte abnormalitie $s$ in children observational national study investigators; 4(11): 1025-1031.

Sagar, T.; Parveen, H.; Hemant, K.; et al., (2016): Radiology of corrosive poisoning: report of three cases. Panacea Journal of Medical Sciences;6(1): 40-43.

Seif, E.; Gomaa, R. and Eisa, M. (2016): A retrospective study of acute poisoning in children under 5 years old admitted to Alexandria poison center in Egypt. World Journal of Preventive Medicine; 4(2): 32-39.

Struck, M.; Beilicke, A.; Hoffmeister, A.; et al., (2016): Acute emergency care and airway management of caustic ingestion in adults: single center observational study. Scand J Trauma Resusc Emerg Med; 24: 45.

Suber, R. (1994): Clinical pathology methods for toxicology. In: Principles and Methods of Toxicology, Hayes AW (ed.) $3^{\text {rd }}$ ed., Raven Press, New York; PP: 476-496.

Sudarsi, B.; Rani, K.; Siddeswari, R.; et al., (2015): Clinical and endoscopic study of upper GI manifestation in corrosive acid ingestion. International Journal of Scientific and Research Publications, 5 (2):1-5.

Taylor, J. (1990): Statistical technique for data analysis. $2^{\text {nd }}$ ed., Lewis Pub. Inc., USA, PP: 25-30.

Urganci, N.; Usta, M.; Kalyoncu, D.; et al., (2014): Corrosive substance ingestion in children. Indian J Pediatr; 81(7):675-679.

Vezakis, A.; Pantiora, E.; Kontis, E.; et al., (2016): Clinical spectrum and management of caustic ingestion: a case series presenting three opposing outcomes. Am J Case Rep; 17: 340-346.

Wightman, R.; Read, K. and Hoffman, R. (2016): Evidence-based management of caustic exposures in the emergency department. Emerg Med Pract;18(5):1-17. 
القيمه التنبوئه لبصض المظاهر الإكلينيكيه والمعايير الكيميائيه الحيويه المبلئيه لتقييم النتيجه فى الأطفال المتسممين بالمواد المسبيه للتآكل

\section{إجلال حسن العوضى 1 ـ رباب نبيل حافظا ــ ميرهان احمد نصر 2

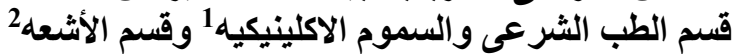

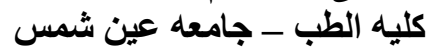

إن إبتلاع منتجات المواد المسبيه للتآكل في الأطفال يشكل مظاهر إكلينيكيه محتمله شديدة السوء و مضاعفات لمدى الحياة و التي تظل واحده من التحديات الصعبه خلال الممارسه الطبيه. ويعتبر التتخيص السريع و التنبير العلاجى المناسب ذو أهميه قصوى فى تقليل الوفيات و الوصول لنتائج مناسبه على المدى الطويل ـ الهدف من الدراسة : التحقيق فى إمكانية التنبؤ بثدة النتيجه للتسمم

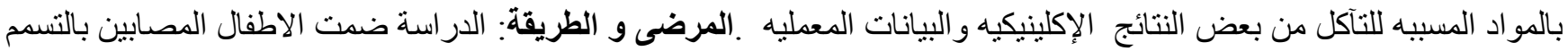

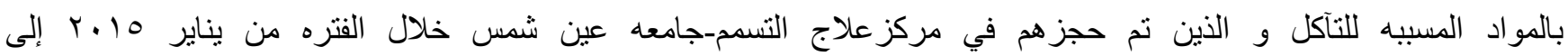
سبتمبر 1 ـ ـ، حيث تم تسجيل المتغيرات الديموجر افيه و النتائج الإكلينيكيه عند الدخول وكذلك البيانات المعطليه الروتينيه ومتغيرات النتيجه (مده الإقامه بالمستشفى ،حدوث التضيق و معدل الوفيات). تم تقسيم المرضى إلى مجمو عتين :مجمو عه عدم وجود مضاعفات و

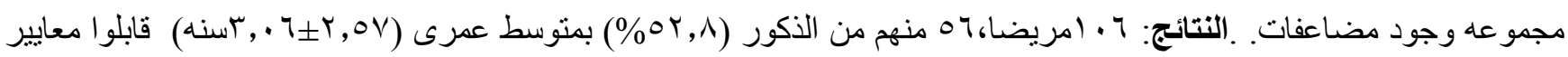
الإدراج ـ و قد وجدت زياده ذات دلاله احصائيه فى العلامات و الأعراض مثل ( القىء و صعوبه البلع وسيلان اللعاب و القىء الدموى و صعوبه التنفس) فى مجمو عه وجود مضاعفات عند مقارنتها بمجمو عه عدم وجود مضاعفات ،بينما لم يكن هناك فروق ذات التات دلاله احصائيه بين الهجمو عتين فيما يخص الإصابات الفميه و الصرير التنفسى. كما لوحظ وجود إرتباط طردى ذو دلاله احصائيه بين النسب الأوليه للقيء و سيلان اللعاب وصعوبه البلع و القىء الدموى، ولم يظهر هذا الإرتباط مع صعوبه التنفس، وبين سوء النتيجه

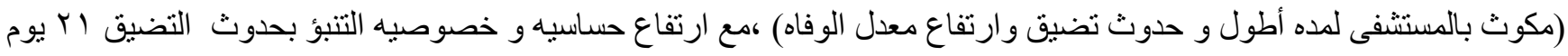

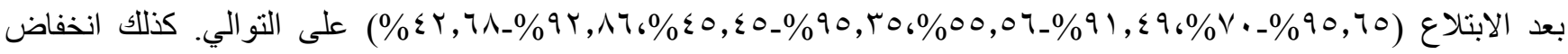
مستويات الاس الهيدروجينى و الهيموجلوبين كان اكثر وضوحا فى مجموعة حدوث مضاعفات وكان له إرتباط طردى ذو دلاله

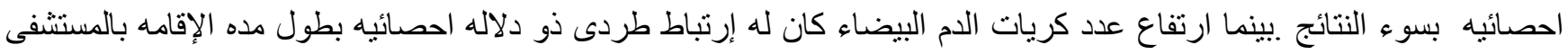

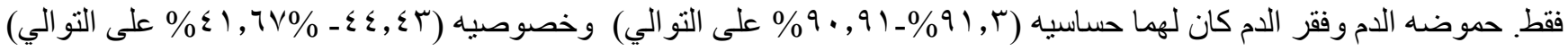
للتنبؤ بحدوث التضيق. الخلاصه: فى حالة إبتلاع المواد المسببه للتآكل فى الأطفال، بعض المظاهر الإكلينيكيه مثل القىء و سيلان اللعاب وصعوبه البلع والقىء الدموى مع وجود البيانات المعطليه مثل إنخفاض الاس الهيدروجينى و الهيموجلوبين و إرتفاع عدد كريات الدم البيضاء عند الدخول ،كانوا أكثر وضوحا فى الحالات ذات المضاعفات كما لوحظ أنهم يمثلوا عوامل تتبؤيه لثنده النتيجه بمكن

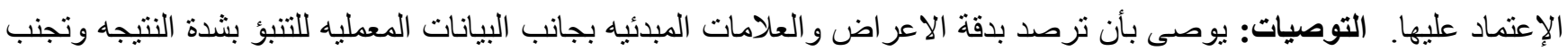

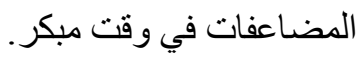

